\title{
A Novel Skull Stripping and Enhancement Algorithm for the Improved Brain Tumor Segmentation using Mathematical Morphology
}

\author{
Benson C. C. and Lajish V. L. \\ Department of Computer Science, University of Calicut, Kerala, India- 673635 \\ E-mail: \{benson_dcs@uoc.ac.in, lajish@uoc.ac.in\} \\ Kumar Rajamani \\ Robert Bosch Engineering and Business Solutions Bangalore, India -560 095 \\ E-mail: kumartr@gmail.com
}

\begin{abstract}
Human brain is a complex system, made up of neurons and glial cells. Nothing in the universe can compare with the functioning of human brain. Due to its complex nature, the diseases affected on the brain is also very complex in nature. Brain imaging is the widely used method for the diagnosing of such deceases. Brain tumor is an abnormal mass of tissue in which cells grow and multiply uncontrollably, seemingly unchecked by the mechanisms that control normal cells. Magnetic Resonance Imaging (MRI) is a commonly used modality for detecting the brain diseases. In this work we proposed a novel method for the preprocessing of MR brain images for the improved segmentation of brain tumor based on mathematical morphology operations. The first part of this paper proposes an efficient method for the skull stripping of brain MR images based on mathematical morphology. One of the main disadvantages of MRI technology is its low contrast. The second part of this paper implements an algorithm for the contrast enhancement of MR brain images using morphological operations. The output of this algorithms are evaluated using standard measures. The experimental part shows that the proposed method produces very prominent and efficient results.
\end{abstract}

Index Terms - Contrast Enhancement, Skull Stripping, Mathematical Morphology, Erosion, Dilation.

\section{INTRODUCTION}

Human brain is the most important and highly organized structure of human body. It coordinates and controls all the complex activities in the body. Brain and spinal cord makes the central nervous system (CNS). The average weight of human brain is about 3 pounds and is made up of more than 100 billion neurons. Human brain is made up of two types of tissues- gray matter and white matter. Gray matter contains unmyelinated neurons, most of which are interneurons. White matter is made up of bundles of long-range myelinated neurons. Brain and spinal cord baths in a particular type of fluid called cerebrospinal fluid (CSF). CSF is responsible for the protection of brain which helps to cushion any physical blows to the head. The brain is covered by a three layered structure named as Meninges. The inner layer of meninges is called pia mater, which is closest to the brain. The middle layer is called arachnoid. The outermost layer of meninges is called dura matter. Human brain is protected with a strong covering called as skull, which is made up of several bones [1-3].

Brain tumor is the abnormal growth of cells in the brain or the membranes surrounding the brain. There are about more than 100 different types of brain tumors discovered [4]. Diagnosing of brain tumor is a challenging task since the anatomical structure of brain is very complex. Recently there are many types of medical imaging modalities available for the diagnosing purposes.

Medical imaging is the process of representing internal structure of human body for various clinical purposes such as medical procedures, diagnosing, surgery planning, and study of normal and abnormal parts etc. The concept of medical imaging was invented by W. C. Rontgen in 1895. He accidently discovered the X-rays, which helps to picturize the internal parts of human body in different shades of black and white [5]. After the invention of XRays, different anatomical and functional medical imaging modalities are emerged for medical imaging. MRI is one of the commonly used methods for brain imaging. MRI works on the basis of principle of nuclear magnetic resonance (NMR). It is a non-invasive imaging method and mainly used for the representation of soft tissues. The main advantage of MRI over other imaging modalities is that it does not emit any harmful radiations to the human body [6].

Figure. 1 represents an example of MR axial plane image of human brain. Though skull is an important part of human brain, it is not required for the analysis purposes [7]. So we need to remove the skull and other non-cerebral structures from MR brain images. That is, we need to extract the non-cerebral parts such as skull, Cerebrospinal Fluid (CSF) and Meninges. Skull stripping is an important pre-processing stage in the analysis of MR images. More accurate skull stripping algorithm 
reduces the probability of misclassification of abnormal tissues [8]. Here we proposes a novel algorithm for the skull stripping based on the technology of mathematical morphology.

One of the main problems associated with the MR images is its low contrast. It is due to the large amount of water content present in the human body. For the better visual quality, we need to increase the magnetic power of the MRI machine. But it is harmful to the human body. The other solution is to use some efficient algorithms for the contrast enhancement [9]. In this paper we proposes an efficient method for the contrast enhancement of MRI brain images based on the idea of mathematical morphology.

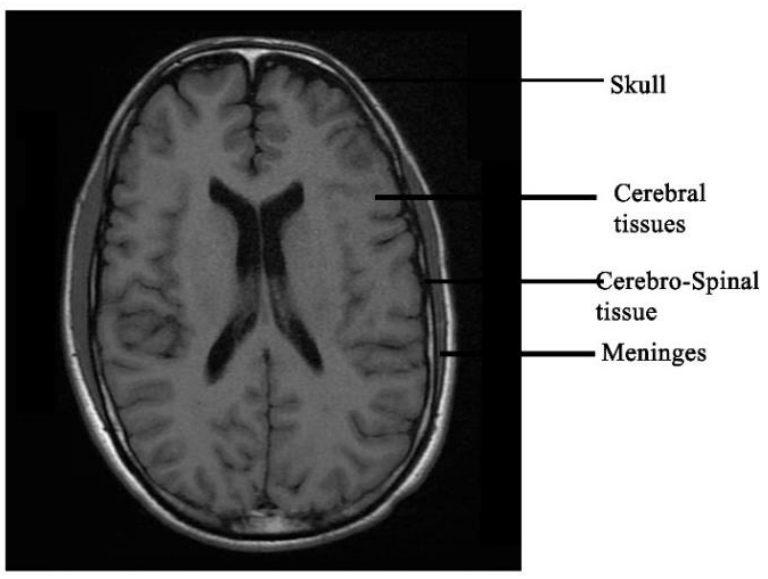

Fig.1. Axial Plane of Human Brain

The rest of the paper is organized as follows. Next section describes the literature survey. Section III presents the materials and methods used for this work. Fourth section covers the experimental results and discussions. And finally last section includes the conclusions and future enhancements.

\section{RELATED WORKS}

Brain imaging is a widely used method for detecting many abnormalities such as tumor, stroke, paralysis, Alzheimer's etc. MRI is one of the most efficient methods for brain scanning. The main problem related with MRI brain images is the presence of skull in the images. For the accurate segmentation of brain tissues, skull must be removed initially. Many methods are proposed for the extraction of brain portion from the MR brain images. F. Segonne et al. proposed an efficient method for the skull stripping of MR brain images based on a hybrid approach of watershed algorithm and deformable surface models [10]. They used the advantage of robustness of the watershed algorithm and the surface information availability advantage of the deformable surface models. Watershed algorithm is used to build an initial approximation of the brain and surface deformation method produces accurate skull stripped images. Finally they compared the results with other publicly available skull stripping tools.
Andre G.R. Balan et al. implemented a novel method for the skull stripping of $3 \mathrm{D}$ MR brain images using a method called Human Encephalon Automatic Delimiter (HEAD) [11]. Their algorithm includes two stages- first stage includes the process of removing background and the second stage includes the process of extracting brain region. For the background removal they used gray level histogram of the images and for the brain extraction they used combination of thresholding and morphological operations.

Juan Eugenio Iglesias et al. introduced a robust, learning based brain extraction system (ROBEST) for the skull stripping from MR brain images [12]. In this method they combined a discriminative model called random forest classifier and a generative model called point distribution model for the skull stripping. Random forest classifier is used to detect the brain boundary and the point distribution model is used to ensure the result is plausible. Finally they proved that their ROBEST method produced more prominent result than the other wellknown skull stripping methods. Francisco J. Galdames et al. proposed another efficient method for the skull stripping based on deformable models and histogram analysis. Their method is called Simplex Mesh and Histogram Analysis Skull Stripping (SMHASS) [13]. They applied a pre-processing method for finding the optimal starting point for the deformation.

Audrey H. Zhuang et al. implemented a mathematical algorithm for the skull stripping on the basis of model based level set (MLS) [14]. The level set method is used to evolve an active curve. The evolution of active curve controlled by two terms in the level set equation. One is developed from the mean curvature of the curve and the second one is designed to model the intensity properties of the cortex in MR images. Orazio Gambino et al. proposed an automatic algorithm for the skull stripping using morphological operators and fuzzy c-means algorithm [15]. Morphological opening operator is used to separate the brain region from the non-brain part. Fuzzy c-means algorithm is used to identify the background and the foreground of each transversal slice. Finally they compared the results with those obtained using the MRIcro software. Dwarikanath Mahapatra suggested a novel technique for the skull stripping of MR brain images using the prior shape information [16]. The prior shape information is computed from a set of labeled training images.

Mathematical morphology is the most commonly used method for skull stripping from MR brain images. In the literature we can see many methods for the separation brain and non-brain tissues based on the concept of mathematical morphology [17-20]. One of the main problems associated with these methods is that it may not work with the different MRI sequences. In this work we proposed an efficient method for the skull stripping on the basis of mathematical morphology, which is compatible with different MRI sequences such as T1, T2, FLAIR, and DWI.

Another important disadvantage associated with the MRI is that it produces low contrast images. For accurate 
diagnosing, finer details in the image should be visible. Therefore contrast enhancement is an important preprocessing step in the area of brain image analysis [21]. In the literature we can see many methods for the contrast enhancement of MR brain images. Most of the methods are based on histogram equalization [22]. Pratik Vinayak Oak et al. implemented and compared various histogram equalization methods for the contrast enhancements of MR brain images [23]. They implemented conventional histogram equalization (HE), bi-histogram equalization (BHE) [24], modified bihistogram equalization (MBHE) [25], brightness preserving $\mathrm{BHE}$ (BBHE) [24], Adaptive histogram equalization (AHE) [26] and contrast limited adaptive histogram equalization (CLAHE) [27]. Finally they concluded that for the better result, selection of the enhancement algorithm should be consider the nature of the images also.

N Senthilkumaran and J Thimmiaraja applied a set of histogram equalization methods on MR brain images [28] They implemented different histogram equalization methods-brightness preserving BHE (BBHE) [24], Recursive Mean-Separate Histogram Equalization (RMSHE) [29], Brightness Preserving Dynamic Histogram Equalization (BPDHE) [30], Dualistic SubImage Histogram Equalization (DSIHE) [31] and Minimum Mean Brightness Error Bi-Histogram Equalization (MMBEBHE) [32].

A. Djerouni et al. proposed an efficient method for the enhancement of MR brain images called Newtonian operator (NO) [33]. After the enhancement they performed segmentation operation using fuzzy clustering method. Their method can be considered as a convolution filter but presents the originality of the adaptive found of convolution mask coefficients. Experimental results shows that their method produces images with low noise susceptibility and the low contrast images are enhanced without over-smoothing the edges.

\section{MATERIALS AND METHODS}

Image pre-processing is an important stage in the medical image processing applications. In medical images, a good pre-processing method will leads to the better segmentation results. So in order to obtain relevant and accurate segmentation results, we have to apply several pre-processing steps. In this paper we proposes two MR brain image pre-processing methods-skull stripping and contrast enhancement. Skull stripping is the process of removing non-brain tissues from the MR brain images. Image enhancement methods inquire about how to improve the visual appearance of images from Magnetic Resonance Image (MRI). Both pre-processing methods are worked on the basis of mathematical morphology. Figure. 2 represents the block diagram of the proposed pre-processing method.

\section{A. MR Image Dataset}

For the experimental purposes we have used a total of 120 samples of MRI brain images (30 T1 images, $30 \mathrm{~T} 2$ images, 30 FLAIR images and 30 DWI images). Images are provided by the Medical College hospital, Thrissur. All the images are taken from the 2D axial plane with the dimension of $512 \times 512$.

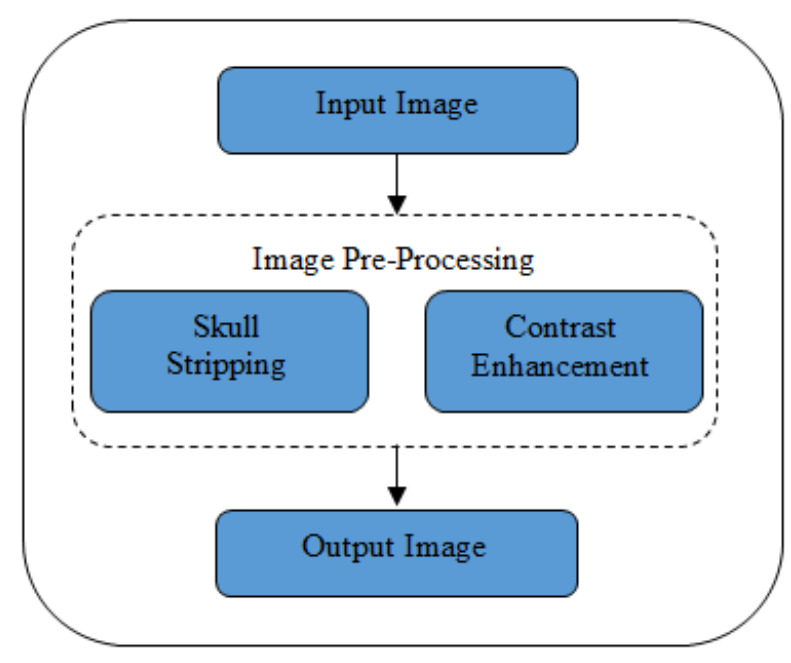

Fig.2. Block Diagram of the Proposed Work

\section{B. Mathematical Morphology}

Mathematical morphology is a shape based tool used to extract the image components which are useful in the representation and description of shape, region, boundaries and convex hull [34]. The concept of mathematical morphology was introduced by Matheron and Serra at the Ecole des Mines in Paris [35]. Mathematical morphology is developed on the basis of set theory. It was initially used for the processing of binary images and later it is applied on the other type of images such as gray scale and color images. The main idea of mathematical morphology is to examine the morphological structure of an image using some small patterns at various locations on the image. These small patterns are called structuring element (SE). A structuring element is a small connected component of pixels. One can be used to extract the useful morphological information from the images by using the structuring elements. The shape and size of the structuring element varies between different applications. Any matrix can be used as a structuring element. There are many known structuring elements. Some of them are diamond, square, disc, horizontal line, vertical line, cross etc. Examples of structuring elements are given in the figure. 3 .

Erosion and dilation are the two fundamental operations in mathematical morphology. Many other operations can be derived by using erosion and dilation. Opening and closing are the examples of such derived operations. Erosion is used to shrink the components of an image and dilation is used to expand the components.

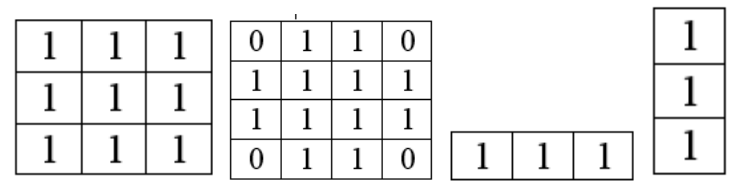

Fig.3. Sample Structuring Elements 
Let $A$ is a set (binary image) and $S$ be the structuring element in $Z^{2}$. Then the Erosion of $A$ by the structuring element $S$, denoted by $A \Theta S$ is the set of all pixels which $S$ placed at that pixel is contained within A. In another way we can say that the Erosion of $A$ by $S$ is the set of all points $z$ such that $S$, translated by $z$, is contained in $A$.

$$
A \Theta S=\left\{z \mid(S)_{z} \subseteq A\right\}
$$

Erosion shrinks or thins the shape of an object. It is also used to exclude the small unwanted objects from the given image.

Dilation of $A$ by $S$ in $Z^{2}$, denoted by $A \oplus S$. Dilation of $A$ by the structuring element $S$ the set of all displacements $z$, such that $\hat{S}$ and $A$ overlap by at least one element.

$$
\mathrm{A} \oplus \mathrm{S}=\left\{\mathrm{z} \mid(\hat{S})_{\mathrm{z}} \cap \mathrm{A} \neq \varnothing\right\}
$$

Dilation is used for growing or thickening an image. It is also used to highlight the small objects in an image.

Opening and Closing are the other two operations in the mathematical morphology. Opening operation makes the contour of an object smoothened and break the narrow lines. Similarly closing operation also smoothens the contours, but it usually eliminates discontinuity and small gaps between the objects. Opening operation is denoted by $A \circ S$ and closing operation is denoted by $A \bullet S$, where $A$ is the input image and $S$ is the structuring element. In opening erosion is performed followed by dilation and in closing dilation is performed followed by erosion.

$$
\begin{aligned}
& \text { Opening, } \mathrm{A} \circ \mathrm{S}=(\mathrm{A} \Theta \mathrm{S}) \oplus \mathrm{S} \\
& \text { Closing, } \mathrm{A} \bullet \mathrm{S}=(\mathrm{A} \oplus \mathrm{S}) \Theta \mathrm{S}
\end{aligned}
$$

Another important idea used in the mathematical morphology is morphological reconstruction. Using morphological reconstruction we can extract some connected components of an image, which are marked in the image. Morphological reconstruction can be implemented by applying series of dilation operations, until the edge of the marker image fits under a second image, which is called the mask image.

\section{Skull Stripping}

Automatic skull stripping from MR brain images is one of the important phase in the neuro-image analysis. The accuracy of the skull stripping algorithm affects many applications such as tumor segmentation, cortical surface reconstruction, pre-surgical planning etc. It is the process of removing non-cerebral tissues such as skull, meninges and eyeball from the MR brain images. In this paper we proposes an automatic skull stripping method based on mathematical morphology.

Working of the skull stripping algorithm is detailed below. The input MR brain image is binarized using Otsu's method [36]. Otsu's method finds the threshold that minimizes within-class variance between two different classes. From the binarized image, largest connected component is found. Brain part will be the largest connected component in the image. The largest connected component is then dilated with a $3 \times 3$ square structuring element so as to preserve minute brain information in the output image. The holes in the resultant image is filled to make the brain a complete connected component. The resulting pixels are superimposed with the input image for getting the skull stripped image.

The pseudo-code of the skull stripping algorithm is given below. Input of the algorithm is MR brain image, im and output is the skull stripped image, img stripped.

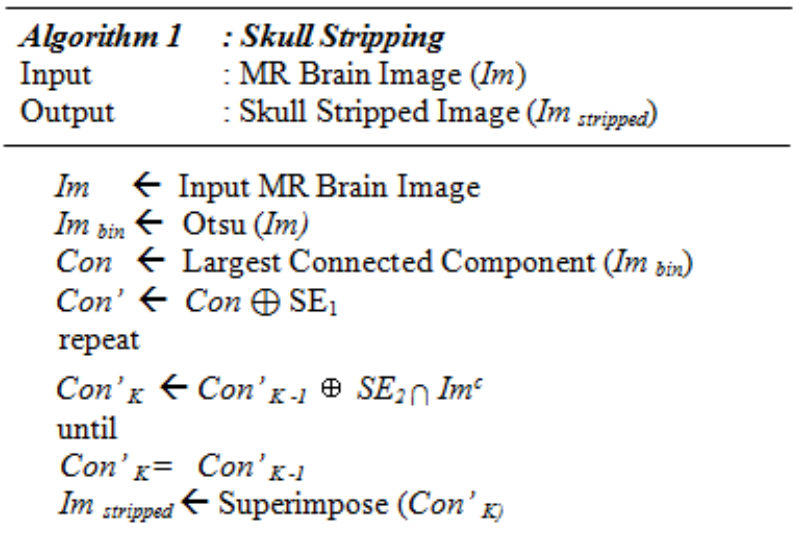

\section{Contrast Enhancement}

MR images are usually low contrast images. To make the images more visual, a hardware or software level image enhancement should be performed. Contrast enhancement is the process of improving visual components of an image so that it is suitable for certain applications. It helps to enhance the clarity of the image, which makes further analysis becomes easier and faster. Enhancement also explores the hidden features in the image. One of the commonly used methods for MR image enhancement is histogram equalization. The main problem associated with the histogram equalization is that it may not be works with all sequence of MR images. Here we proposes an efficient method for the contrast enhancement based on mathematical morphology. Since the structure of the brain is round shaped, here we used a disc structuring element for morphological operations.

Working of contrast enhancement algorithm is explained as follows. Initially algorithm finds the complement of given skull stripped MR brain image. Closing operation is applied on the complemented image using disk structuring element. Then calculates the complement of previous result. Later the mathematical operation finds the difference between the original image and resultant image. Finally, original image is added with the differenced image to get the final contrast enhanced image.

The pseudo-code of the contrast enhancement algorithm is given below. Input of the algorithm is skull stripped MR brain image, Im stripped and output is the enhanced image, Img enhanced. 


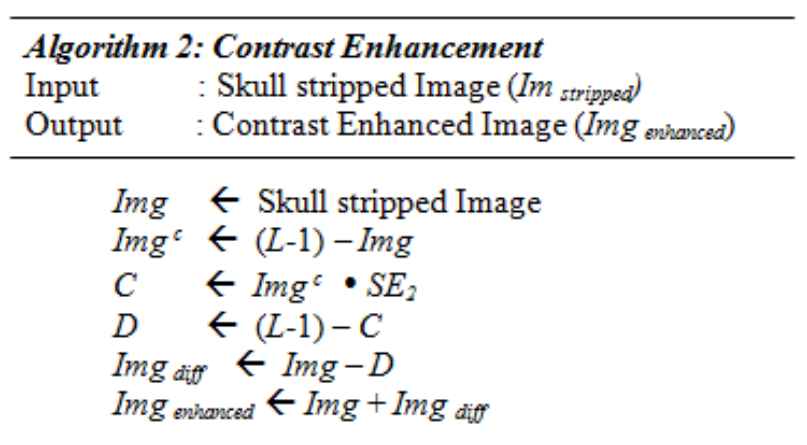

\section{RESULTS AND DISCUSSIONS}

Skull stripping and contrast enhancement are the two important pre-processing stages in tumor characterization. It helps to avoid non brain information from the input image and to enhance the contrast of the image. Figure. 4-7 shows the results of the proposed work on different MR Brain Image sequences. The algorithms are applied on the various image sequences of brain MRI. First column represents the input MR images, second column represents the skull stripped images and last column includes the contrast enhanced images.

Result of the skull stripping algorithm is compared with the manually marked ground truth using two standard similarity coefficients Dice [37] and Jaccard [38]. Skull stripping is carried out by using Matlab toolkit and the ground truth is created with the help of ITK Snap tool [39]. It is an open source software that contains innovative tools for manual outlining and quality control. The performance of the skull stripping algorithm is given in the table. 1. The false positive rate (FPR) and false negative rate (FNR) are also included in the table. FPR shows the degree of under segmentation and FNR shows degree of over segmentation. Average performance using Dice coefficient is 94.33 and using Jaccard coefficient is 90.88. False positive rate is 0.0061 and false negative rate is 0.0423 .

$$
\begin{aligned}
\text { Dice Coefficient } & =\frac{2|A(S) \cap A(G)|}{|A(S)|+|A(G)|} \\
& =\frac{T P}{T P+F P+F N} \\
\text { Jaccard Coefficient } & =\frac{A(S) \cap A(G)}{A(S) \mathrm{U} A(G)} \\
& =\frac{2 T P}{2 T P+F P+F N} \\
\mathrm{FPR} & =\frac{F P}{T N+F P} \\
\mathrm{FNR} & =\frac{F N}{T P+F N}
\end{aligned}
$$

$\mathrm{A}(\mathrm{S})=$ Pixels calculated using the proposed algorithm $A(G)=$ Pixels obtained from the ground truth $\mathrm{TP}=$ Pixels that are correctly classified as brain tissue $\mathrm{FP}=$ Pixels that are incorrectly classified as brain tissue

$\mathrm{TN}=$ Pixels that are correctly classified as non-brain tissue

$\mathrm{FN}=$ Pixels that are incorrectly classified as non-brain tissue

Table 1. Performance of Skull Stripping Algorithm

\begin{tabular}{|c|c|c|c|c|}
\hline & Dice & Jaccard & FPR & FNR \\
\hline DWI & 96.18 & 92.12 & 0.0054 & 0.0291 \\
\hline FLAIR & 95.61 & 91.33 & 0.0059 & 0.0328 \\
\hline T1 & 93.08 & 90.13 & 0.0063 & 0.0481 \\
\hline T2 & 92.45 & 89.95 & 0.0069 & 0.0594 \\
\hline $\begin{array}{c}\text { Average } \\
\text { Performance }\end{array}$ & $\mathbf{9 4 . 3 3}$ & $\mathbf{9 0 . 8 8}$ & $\mathbf{0 . 0 0 6 1}$ & $\mathbf{0 . 0 4 2 3}$ \\
\hline
\end{tabular}

The efficiency of the contrast enhancement algorithm is calculated using some standard measures such as Signal to Noise ratio (SNR), Peak signal to Noise Ratio (PSNR), Root Mean Square Error (RMSE) and Mean Absolute Error (MAE) [40]. The performance of the enhancement algorithm is given in the table. 2. Average value of Signal to noise ratio is 23.67 , peak signal to noise ratio is 27.10 , root mean square is 11.52 , and mean absolute error is 5.01 .

$$
\begin{gathered}
\text { SNR }=10 . \log _{10}\left[\frac{\sum_{0}^{n x-1} \sum_{0}^{n y-1}[r(x, y)]^{\wedge} 2}{\sum_{0}^{n x-1} \sum_{0}^{n y-1}[r(x, y)-t(x, y)]^{\wedge} 2}\right] \\
\text { PSNR }=10 . \log _{10}\left[\frac{m a x(r(x, y))^{2}}{\frac{\sum_{0}^{n x-1} \sum_{0}^{n y-1}[r(x, y)-t(x, y)]^{2}}{n x . n y}}\right] \\
R M S E=\sqrt{\frac{\sum_{0}^{n x-1} \sum_{0}^{n y-1}[r(x, y)-t(x, y)]^{\wedge} 2}{n x . n y}} \\
M A E=\frac{\sum_{0}^{n x-1} \sum_{0}^{n y-1}|r(x, y)-t(x, y)|}{n x . n y}
\end{gathered}
$$

Table 2. Performance of Contrast Enhancement Algorithm

\begin{tabular}{|c|c|c|c|c|}
\hline & SNR & PSNR & RMSE & MAE \\
\hline DWI & 24.58 & 27.47 & 10.79 & 4.83 \\
\hline FLAIR & 23.79 & 29.15 & 11.12 & 4.99 \\
\hline T1 & 23.33 & 26.61 & 11.49 & 5.03 \\
\hline T2 & 22.97 & 25.18 & 12.67 & 5.19 \\
\hline $\begin{array}{c}\text { Average } \\
\text { Performance }\end{array}$ & $\mathbf{2 3 . 6 7}$ & $\mathbf{2 7 . 1 0}$ & $\mathbf{1 1 . 5 2}$ & $\mathbf{5 . 0 1}$ \\
\hline
\end{tabular}

The experimental results show that the proposed skull stripping and contrast enhancement algorithm can be effectively used for various medical image analysis applications such as tumor segmentation, classification and characterization. 


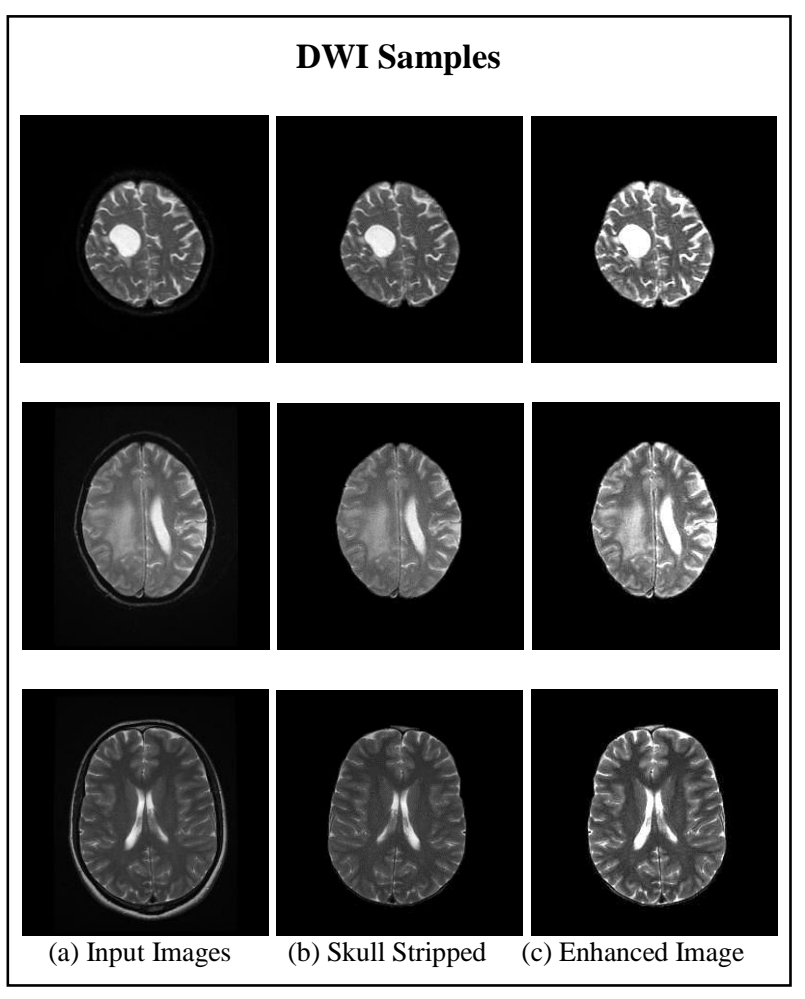

Fig.4. Result on DWI Image Samples

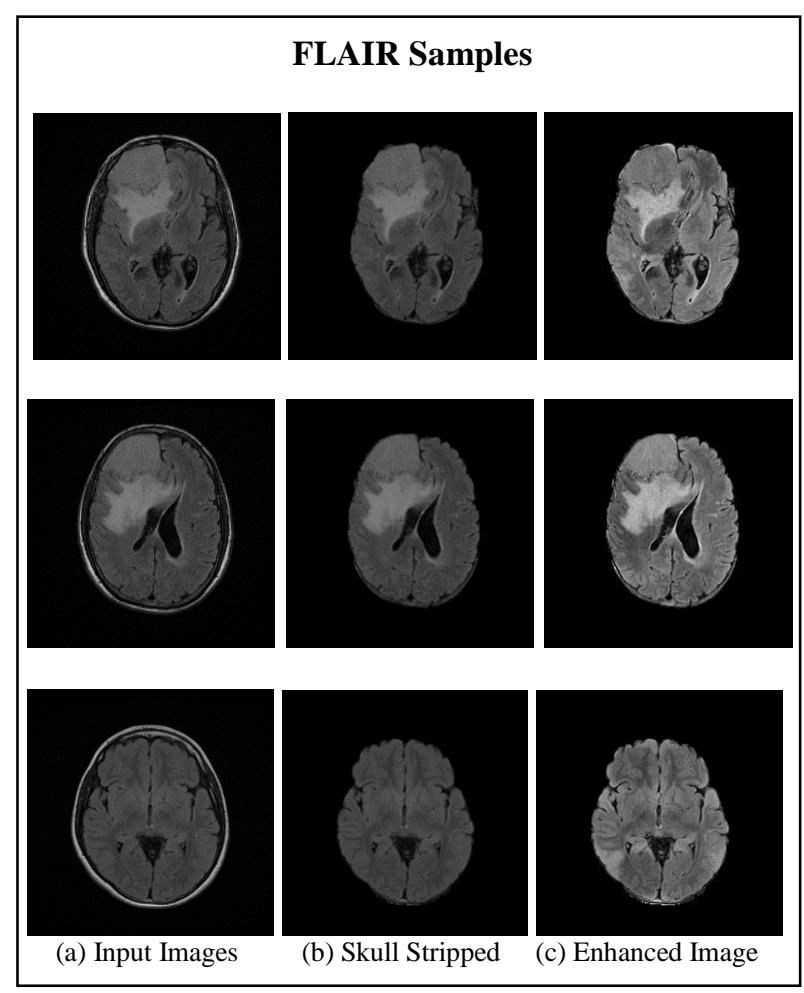

Fig.5. Result on FLAIR Image Samples

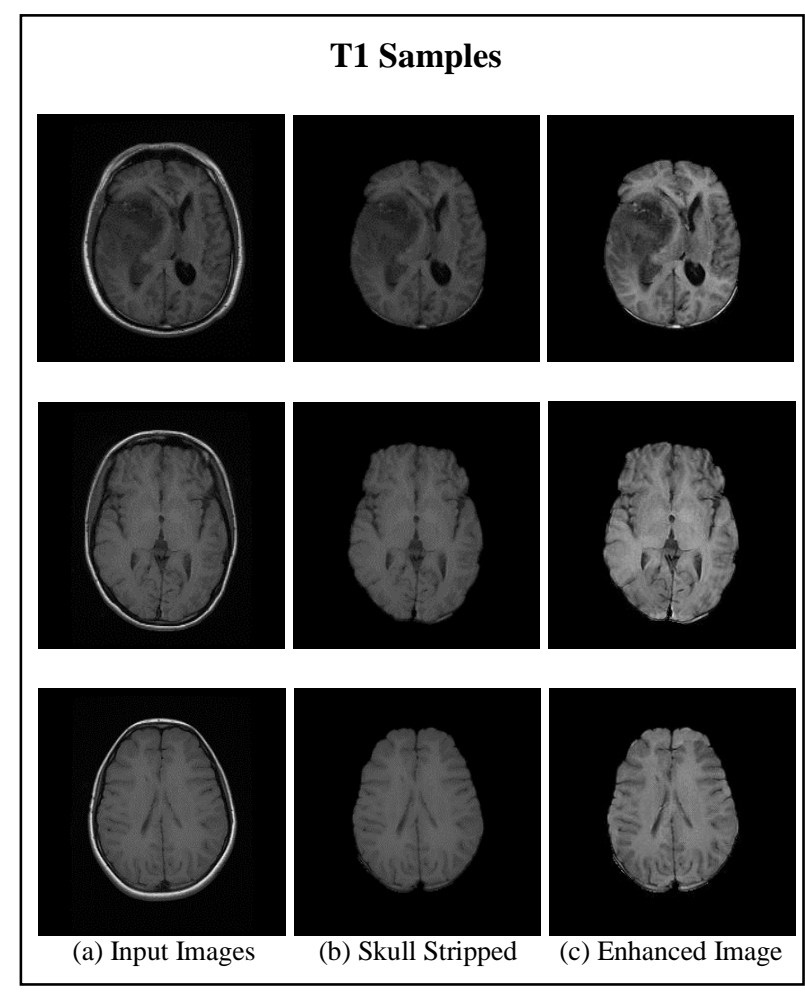

Fig.6. Result on T1 Image Samples

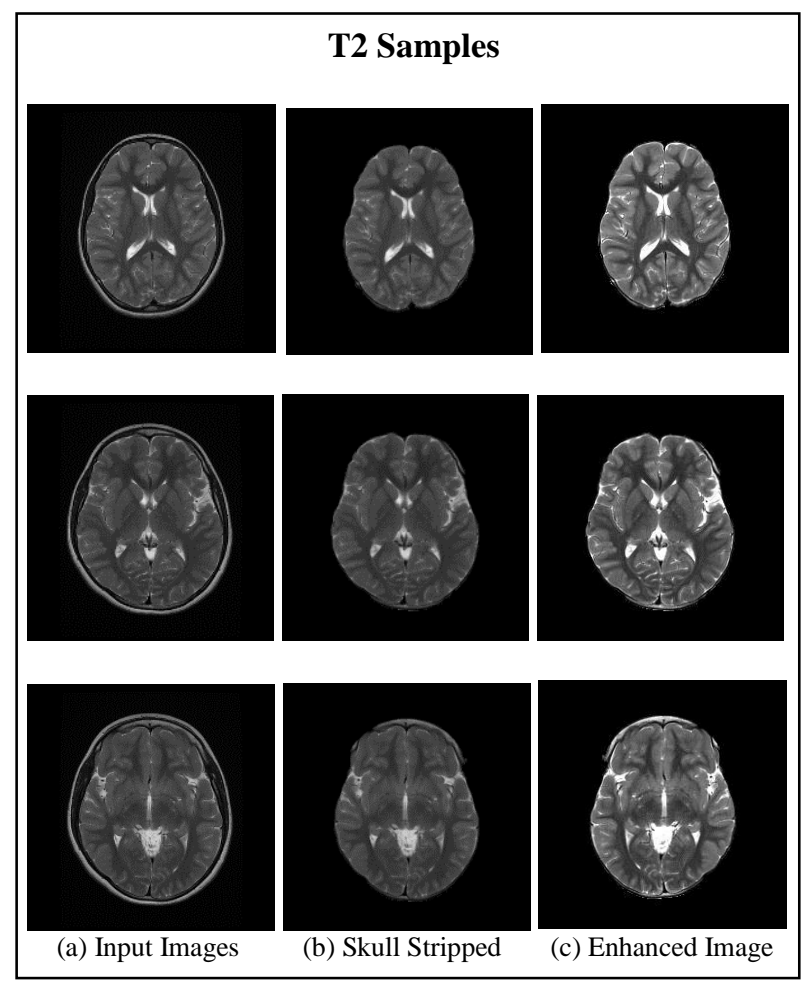

Fig.7. Result on T2 Image Samples 


\section{CONCLUSIONS}

Pre-processing is an important stage in the image processing especially in the case of medical image segmentation applications. An efficient image preprocessing algorithm will increase accuracy of the segmentation algorithms and reduces misclassification. In this paper we propose an efficient method for preprocessing of MR brain images based on mathematical morphological operations. Skull stripping and contrast enhancement are the two pre-processing methods proposed in this paper. Experimental results show that these two pre-processing steps increase the performance of tumor segmentation algorithms. The proposed work can be effectively applied on different MR Image sequences such as DWI, FLAIR, T1 and T2. Now the algorithm worked on the $2 \mathrm{D}$ MR Brain images. In the future we can evolve the algorithms for the preprocessing of 3D MR Brain images.

\section{REFERENCES}

[1] Danielle S. Bassett and Michael S. Gazzaniga, "Understanding complexity in the human brain", Trends in Cognitive Sciences, 15(5), 2011.

[2] Alan Wee-Chung Liew and Hong Yan, "Current Methods in the Automatic Tissue Segmentation of 3D Magnetic Resonance Brain Images", Current Medical Imaging Reviews, 2 (1), 2006.

[3] W.L. Nowinski, "Biomechanics of the Brain" Biological and Medical Physics, Biomedical Engineering”, K. Miller (ed.), Chapter 2, 2011.

[4] S. Cha, "Update on Brain Tumor Imaging: From Anatomy to Physiology", American Journal of Neuroradiology, 27, 2006, 475-487.

[5] https://explorable.com/wilhelm-conrad-roentgen, Wilhelm Conrad Roentgen and the Discovery of X-Ray Beams.

[6] P.Narendran, V. K. Narendira Kumar and K. Somasundaram, "3D Brain Tumors and Internal Brain Structures Segmentation in MR Images", International Journal of Image, Graphics and Signal Processing, 2012 $1,35-43$.

[7] Horst K. Hahn and Heinz-Otto Peitgen, "The Skull Stripping Problem in MRI Solved by a Single 3D Watershed Transform", Proc. MICCAI, LNCS, 1935, 2000, 134-143, Springer.

[8] Shafaf Ibrahim, Noor Elaiza Abdul Khalid, Mazani Manaf and Mohd Ezane Aziz, "Qualitative Analysis of Skull Stripping Accuracy for MRI Brain Images", Ubiquitous Information Technologies and Applications, Lecture Notes in Electrical Engineering, 2013.

[9] Sonia Goyal and Seema Baghla, "Region Growing Adaptive Contrast Enhancement of Medical MRI Images", Journal of Global Research in Computer Science, 2(7), 2011.

[10] F. Segonne, A.M. Dale, E. Busa, M. Glessner, D. Salat, H.K. Hahn, and B. Fischl, "A hybrid approach to the skull stripping problem in MRI”, NeuroImage, 22, 2004, 1060- 1075 .

[11] Andre G.R. Balan, AgmaJ.M.Traina, Marcela X. Ribeiro, Paulo M. A. Marques and Caetano Traina Jr., "Smart histogram analysis applied to the skull-stripping problem in T1-weighted MRI", Computers in Biology and Medicine, 42, 2012, 509-522.
[12] Juan Eugenio Iglesias, Cheng-Yi Liu, Paul Thompson and Zhuowen $\mathrm{Tu}$, "Robust Brain Extraction across Datasets and Comparison with Publicly Available Methods", IEEE Transactions on Medical Imaging, 2011.

[13] Francisco J. Galdames, Fabrice Jaillet and Claudio A Perez, "An Accurate Skull Stripping Method Based on Simplex Meshes and Histogram Analysis in Magnetic Resonance Images", Elsevier, Journal of Neuroscience Methods, 206, 2012, 103-119.

[14] Audrey H. Zhuang, Daniel J. Valentino and Arthur W. Toga, "Skull-stripping Magnetic Resonance Brain Images Using a Model-based Level Set", Neuro Image, 32, 2006, 79-92.

[15] Orazio Gambino, Enrico Daidone, Matteo Sciortino, Roberto Pirrone and Edoardo Ardizzone, "Automatic Skull Stripping in MRI based on Morphological Filters and Fuzzy C-means Segmentation", 33rd Annual International Conference of the IEEE EMBS Boston, Massachusetts USA, 2011.

[16] Dwarikanath Mahapatra, "Skull Stripping of Neonatal Brain MRI: Using Prior Shape Information with Graph Cuts”, Springer, J Digit Imaging, 25, 2012, 802-814.

[17] Rosniza Roslan, Nursuriati Jamil and Rozi Mahmud, "Skull Stripping of MRI Brain Images using Mathematical Morphology", 2010 IEEE EMBS Conference on Biomedical Engineering \& Sciences (IECBES 2010), 2010.

[18] John Chivertona, Kevin Wells, Emma Lewis, Chao Chen, Barbara Podda and Declan Johnson, "Statistical Morphological Skull Stripping of Adult and Infant MRI Data", Computers in Biology and Medicine, 37, 2007, 342-357.

[19] Selvaraj. D and Dhanasekaran. R. "MRI Brain Tumor Detection by Histogram and Segmentation by Modified GVF Model", International Journal of Electronics and Communication Engineering \& Technology (IJECET), 4(1), 2013, 55-68.

[20] Sajjad Mohsin, Sadaf Sajjad, Zeeshan Malik, and Abdul Hanan Abdullah, "Efficient Way of Skull Stripping in MRI to Detect Brain Tumor by Applying Morphological Operations, after Detection of False Background", International Journal of Information and Education Technology, 2(4), 2012.

[21] Maher un Nisa and Ahsan Khawaja, "Contrast Enhancement Impact on Detection of Tumor in Brain MRI", Science International, (Lahore), 27(3), 2015, 2161-2163.

[22] R. C. Gonzales and R. E. Woods, "Digital Image processing", Third Edition, Prentice Hall, 2008.

[23] Pratik Vinayak Oak and Prof. Mrs. R. S. Kamathe, "Contrast Enhancement of Brain MRI Images using Histogram based Techniques", International Journal of Innovative Research in Electrical, Electronics, Instrumentation and Control Engineering, 1(3), 2013.

[24] Y. T. Kim, "Contrast Enhancement Using Brightness Preserving Bi-Histogram Equation”, IEEE Transactions on Consumer Electronics, 43(1), 1997, 1-8.

[25] Ali Ziaei, Hojatollah Yeganeh, Karim Faez and Saman Sargolzaei, "A Novel Approach for Contrast Enhancement in Biomedical Images Based on Histogram Equalization", IEEE International Conference on Bio-Medical Engineering and Informatics, 2008.

[26] D. J. Ketcham, R. W. Lowe and J. W. Weber, "Image enhancement techniques for cockpit displays", Tech. rep., Hughes Aircraft, 1974. 
[27] A. Boschetti, N. Adami, R. Leonardi and M. Okuda, "High Dynamic Range Image Tone Mapping Based On Local Histogram Equalization", IEEE, 2010.

[28] N Senthilkumaran and J Thimmiaraja, "A Study on Histogram Equalization for MRI Brain Image Enhancement", Proc. of Int. Conf. on Recent Trends in Signal Processing, Image Processing and VLSI, Association of Computer Electronics and Electrical Engineers, 2014.

[29] S.-D. Chen and A. R. Ramli, "Contrast Enhancement using Recursive Mean-Separate Histogram Equalization for Scalable Brightness Preservation", IEEE Transactions on Consumer Electronics, 49(4), 2003), 1301-1309.

[30] H. Ibrahim and N. S. Pik Kong, "Brightness Preserving Dynamic Histogram Equalization for Image Contrast Enhancement", IEEE Transactions on Consumer Electronics, 53(4), 2007, 1752-1758.

[31] Y. Wang, Q. Chen and B. Zhang, "Image Enhancement Based on Equal Area Dualistic Sub-Image Histogram Equalization Method", IEEE Transactions on Consumer Electronics, 45(1), 1999), 68-75.

[32] S.-D. Chen and A. R. Ramli, "Minimum Mean Brightness Error Bi-Histogram Equalization in Contrast Enhancement", IEEE Transactions on Consumer Electronics, 49(4), 2003, 1310-1319.

[33] A. Djerouni, H. Hamada, and N. Berrached, "MR imaging contrast enhancement and segmentation using fuzzy clustering", IJCSI International Journal of Computer Science Issues, 8(4), 2011.

[34] R. C. Gonzales and R. E. Woods, "Digital Image processing", Chapter 9, Third Edition, Prentice Hall, 2008.

[35] Jean. Serra, "Image Analysis and Mathematical Morphology", Academic Press, 1982.

[36] N. Otsu's, "A threshold selection method from gray level histograms", IEEE Transactions on systems, MAN and Cybernetics, 9(1), 1979.

[37] Lee R. Dice, "Measures of the Amount of Ecologic Association between Species", Ecological Society of America, 26(3), 1945, 297-302.

[38] Jaccard, P.: The Distribution of Flora in Alpine Zone, New Phytol, 11(2), 1912, 37-50.

[39] P.A. Yushkevich, J. Piven, H. Cody, S. Ho, J.C. Gee, G. Gerig, "User-guided level set segmentation of anatomical structures with ITK-SNAP", Insight J., 1, 2005.

[40] Keun Jo Jang, Dae Cheol Kweon, Jong-Woong Lee, Jiwon Choi, Eun-Hoe Goo, Kyung-Rae Dong, JaeSeung Lee, Gye Hwan Jin and Sungbo Seo, "Measurement of Image Quality in CT Images
Reconstructed with Different Kernels", Journal of the Korean Physical Society, 58(2), 2011, 334-342.

\section{Authors' Profiles}

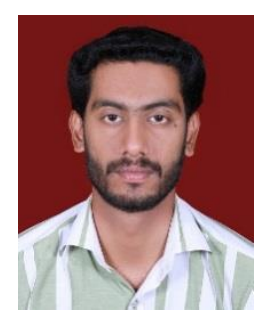

Benson. C. C earned his Masters in Computer Applications from the University of Calicut in 2011 and specialized in Medical Image Processing. $\mathrm{He}$ is an active research scholar working in the area of MR Image analysis under the supervision of Dr. Lajish.V. L.

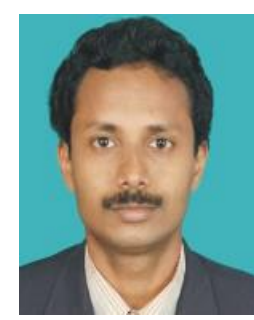

Lajish V.L. has been associated with University of Calicut, Kerala, as Head of the Department of Computer Science. He has worked as Scientist R\&D in TCS Innovation Labs, Tata Consultancy Services Ltd. Mumbai, prior to joining the University. His prime areas of research include Digital Speech and Image Processing, Pattern Recognition algorithms, Indian language script technology solutions for masses. After his Masters in Computer Applications from Vellore Institute of Technology, he earned his Ph.D in Computer Science from University of Calicut, Kerala in 2007. $\mathrm{He}$ is a senior life member of International Association of Computer Science and Information Technology.

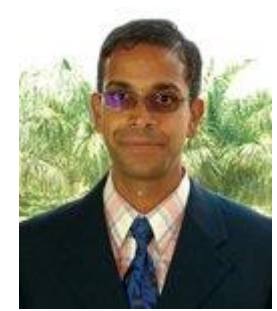

Kumar Rajamani received his MSc (Mathematics) and MTech (CSc) from Sri Sathya Sai Institute of Higher Learning. He received his $\mathrm{PhD}$ degree in Biomedical Engineering from University of Bern, Switzerland. He has three patents to his credit and has published extensively in international journals and conferences $\mathrm{He}$ is currently working as architect at Robert Bosch Engineering and Business Solutions Limited, Bangalore, India. His areas of interests include image processing, medical image analysis and pattern recognition.

How to cite this paper: Benson C. C., Lajish V. L., Kumar Rajamani,"A Novel Skull Stripping and Enhancement Algorithm for the Improved Brain Tumor Segmentation using Mathematical Morphology", International Journal of Image, Graphics and Signal Processing(IJIGSP), Vol.8, No.7, pp.59-66, 2016.DOI: 10.5815/ijigsp.2016.07.07 